Article

\title{
The Extended Minimax Disparity RIM Quantifier Problem
}

\author{
Dug Hun Hong (1)
}

Department of Mathematics, Myongji University, Yongin Kyunggido 449-728, Korea; dhhong@mju.ac.kr

Received: 8 March 2019; Accepted: 1 April 2019; Published: 3 April 2019

\begin{abstract}
An interesting regular increasing monotone (RIM) quantifier problem is investigated. Amin and Emrouznejad [Computers \& Industrial Engineering 50(2006) 312-316] have introduced the extended minimax disparity OWA operator problem to determine the OWA operator weights. In this paper, we propose a corresponding continuous extension of an extended minimax disparity OWA model, which is the extended minimax disparity RIM quantifier problem, under the given orness level and prove it analytically.
\end{abstract}

Keywords: fuzzy sets; RIM quantifier; extended minimax disparity; OWA model; RIM quantifier problem

\section{Introduction}

One of the important topic in the theory of ordered weighted averaging (OWA) operators is the determination of the associated weights. Several authors have suggested a number of methods for obtaining associated weights in various areas such as decision making, approximate reasoning, expert systems, data mining, fuzzy systems and control [1-18]. Researchers can easily see most of OWA papers in the recent bibliography published in Emrouznejad and Marra [5]. Yager [16] proposed RIM quantifiers as a method for finding OWA weight vectors through fuzzy linguistic quantifiers. Liu [19] and Liu and $\mathrm{Da}$ [20] gave solutions to the maximum-entropy RIM quantifier model when the generating functions are differentiable. Liu and Lou [21] studied the equivalence of solutions to the minimax ratio and maximum-entropy RIM quantifier models, and the equivalence of solutions to the minimax disparity and minimum-variance RIM quantifier problems. Hong $[22,23]$ gave the proof of the minimax ratio RIM quantifier problem and the minimax disparity RIM quantifier model when the generating functions are absolutely continuous. He also gave solutions to the maximum-entropy RIM quantifier model and the minimum-variance RIM quantifier model when the generating functions are Lebesgue integrable. Liu [24] proposed a general RIM quantifier determination model, proved it analytically using the optimal control method and investigated the solution equivalence to the minimax problem for the RIM quantifier. However, Hong [11] recently provided a modified model for the general RIM quantifier model and the correct formulation of Liu's result.

Amin and Emrouznejad [1] have introduced the following the extended minimax disparity OWA operator model to determine the OWA operator weights:

$$
\begin{array}{cl}
\text { Minimize } & \max _{i \in\{1, \cdots, n-1\}, j \in\{i+1, \cdots, n\}}\left|w_{i}-w_{j}\right| \\
\text { subject to } & \operatorname{orness}(W)=\sum_{i=1}^{n} \frac{n-i}{n-1} w_{i}=\alpha, 0 \leq \alpha \leq 1, \\
& w_{1}+\cdots+w_{n}=1,0 \leq w_{i}, i=1, \cdots, n .
\end{array}
$$


In this paper, we propose a corresponding extended minimax disparity model for RIM quantifier determination under given orness level and prove it analytically. This paper is organized as follows: Section 2 presents the preliminaries and Section 3 reviews some models for the RIM quantifier problems and propose the extended minimax disparity model for the RIM quantifier problem. In Section 4, we prove the extended minimax disparity model problem mathematically for the case in which the generating functions are Lesbegue integrable functions.

\section{Preliminaries}

Yager [15] introduced a new aggregation technique based on the OWA operators. An OWA operator of dimension $n$ is a function $F: R^{n} \rightarrow R$ that has an associated weighting vector $W=\left(w_{1}, \cdots, w_{n}\right)^{T}$ of having the properties $0 \leq w_{i} \leq 1, \quad i=1, \cdots, n, w_{1}+\cdots+w_{n}=1$, and such that

$$
F\left(a_{1}, \cdots, a_{n}\right)=\sum_{i=1}^{n} w_{i} b_{i}
$$

where $b_{j}$ is the $j$ th largest element of the collection of the aggregated objects $\left\{a_{1}, \cdots, a_{n}\right\}$. In [15], Yager defined a measure of "orness" associated with the vector $W$ of an OWA operator as

$$
\operatorname{orness}(W)=\sum_{i=1}^{n} \frac{n-i}{n-1} w_{i}
$$

and it characterizes the degree to which the aggregation is like an or operation.

The RIM quantifiers was introduced by Yager [16] as a method for obtaining the OWA weight vectors via fuzzy linguistic quantifiers. The RIM quantifiers can provide information aggregation procedures guided by a dimension independent description and verbally expressed concepts of the desired aggregation.

Definition 1 ([14]). A fuzzy subset $Q$ is called a RIM quantifier if $Q(0)=0, Q(1)=1$ and $Q(x) \geq Q(y)$ for $x>y$.

The quantifier for all is represented by the fuzzy set

$$
Q_{*}(r)= \begin{cases}1, & x=1 \\ 0, & x \neq 1\end{cases}
$$

The quantifier there exist, not none, is defined as

$$
Q^{*}(r)= \begin{cases}0, & x=0 \\ 1, & x \neq 0\end{cases}
$$

Both of these are examples of RIM quantifier. To analyze the relationship between OWA and RIM quantifier, a generating function representation of RIM quantifier was proposed.

Definition 2. For $f(t)$ on $[0,1]$ and a RIM quantifier $Q(x), f(t)$ is called generating function of $Q(x)$, if it satisfies

$$
Q(x)=\int_{0}^{x} f(t) d t
$$

where $f(t) \geq 0$ and $\int_{0}^{1} f(t) d t=1$.

If $Q(x)$ is an absolutely continuous function, then $f(x)$ is a Lesbegue integrable function; moreover, $f(x)$ is unique in the sense of "almost everywhere" in abbreviated form, a.e. 
Yager extended the orness measure of OWA operator, and defined the orness of a RIM quantifier [16].

$$
\operatorname{orness}(Q)=\int_{0}^{1} Q(x) d x=\int_{0}^{1}(1-t) f(t) d t .
$$

As the RIM quantifier can be seen as the continuous form of OWA operator with generating function, OWA optimization problem is extended to the RIM quantifier case.

The definitions of essential supremum and essential infimum [21] of $f$ are as follows:

$$
\begin{aligned}
& \text { ess } \operatorname{supf}=\inf \{t:|\{x \in[0,1]: f(x)>t\}|=0\}, \\
& \text { ess inff }=\sup \{t:|\{x \in[0,1]: f(x)<t\}|=0\},
\end{aligned}
$$

where $|E|$ is the Lebesgue measure of the Lebesgue measurable set $E$.

\section{Models for the RIM Quantifier Problems}

Fullér and Majlender [8] proposed the minimum variance model, which minimizes the variance of OWA operator weights under a given level of orness. Their method requires the proof of the following mathematical programming problem:

$$
\begin{array}{ll}
\text { Minimize } & D(W)=\frac{1}{n} \sum_{i=1}^{n-1}\left(w_{i}-\frac{1}{n}\right)^{2} \\
\text { subject to } & \operatorname{orness}(W)=\sum_{i=1}^{n} \frac{n-i}{n-1} w_{i}=\alpha, 0 \leq \alpha \leq 1, \\
& w_{1}+\cdots+w_{n}=1,0 \leq w_{i}, i=1, \cdots, n .
\end{array}
$$

Liu $[19,24]$ extended the minimum variance problem for OWA operator to the RIM quantifier problem case:

$$
\begin{array}{ll}
\text { Minimize } & D_{f}=\int_{0}^{1} f^{2}(r) d r-1 \\
\text { subject to } & \int_{0}^{1} r f(r) d r=1-\alpha, 0<\alpha<1, \\
& \int_{0}^{1} f(r) d r=1, f(r) \geq 0 .
\end{array}
$$

Wang and Parkan [13] proposed the minimax disparity problem as follows:

$$
\begin{array}{cl}
\text { Minimize } & \max _{i \in\{1, \cdots, n-1\}}\left|w_{i}-w_{i+1}\right| \\
\text { subject to } & \operatorname{orness}(W)=\sum_{i=1}^{n} \frac{n-i}{n-1} w_{i}=\alpha, 0 \leq \alpha \leq 1, \\
& w_{1}+\cdots+w_{n}=1,0 \leq w_{i}, i=1, \cdots, n .
\end{array}
$$

Similar to the minimax disparity OWA operator problem, Hong [11] proposed the minimax disparity RIM quantifier problem as follows:

$$
\begin{array}{ll}
\text { Minimize } & \operatorname{ess~sup}_{t \in[0,1]}\left|f^{\prime}(t)\right| \\
\text { subject to } & \int_{0}^{1} r f(r) d r=1-\alpha, 0<\alpha<1 \\
& \int_{0}^{1} f(r) d r=1, \text { absolutely continuous } f(r) \geq 0 .
\end{array}
$$


Wang et al. [14] have introduced the following least squares deviation (LSD) method as an alternative approach to determine the OWA operator weights.

$$
\begin{array}{cl}
\text { Minimize } & \sum_{i=1}^{n-1}\left(w_{i}-w_{i-1}\right)^{2} \\
\text { subject to } & \operatorname{orness}(W)=\sum_{i=1}^{n} \frac{n-i}{n-1} w_{i}=\alpha, 0 \leq \alpha \leq 1, \\
& w_{1}+\cdots+w_{n}=1,0 \leq w_{i}, i=1, \cdots, n .
\end{array}
$$

Hong [25] proposed the following corresponding least squares disparity RIM quantifier problem under a given orness level:

$$
\begin{array}{ll}
\text { Minimize } & D_{f}=\int_{0}^{1}\left(f^{\prime}\right)^{2}(r) d r \\
\text { subject to } & \int_{0}^{1}(1-r) f(r) d r=\alpha, 0<\alpha<1, \\
& \int_{0}^{1} f(r) d r=1, \\
& f(r)>0 .
\end{array}
$$

Recently, Amin and Emrouznejad [1] proposed a problem of minimizing the maximum disparity of any distinct pairs of weights instead of adjacent weights. that is:

$$
\begin{array}{cl}
\text { Minimize } & \max _{i \in\{1, \cdots, n-1\}, j \in\{i+1, \cdots, n\}}\left|w_{i}-w_{j}\right| \\
\text { subject to } & \operatorname{orness}(W)=\sum_{i=1}^{n} \frac{n-i}{n-1} w_{i}=\alpha, 0 \leq \alpha \leq 1, \\
& w_{1}+\cdots+w_{n}=1,0 \leq w_{i}, i=1, \cdots, n .
\end{array}
$$

We consider the following easy important fact.

\section{Note}

$$
\max _{i \in\{1, \cdots, n-1\}, j \in\{i+1, \cdots, n\}}\left|w_{i}-w_{j}\right|=\max w_{i}-\min w_{i} .
$$

For this, first it is trivial that

$$
\max _{i \in\{1, \cdots, n-1\}, j \in\{i+1, \cdots, n\}}\left|w_{i}-w_{j}\right| \leq \max w_{i}-\min w_{i} .
$$

Next, suppose that $\max w_{i}=w_{i_{0}}$, min $w_{i}=w_{j_{0}}$. If $i_{0}<j_{0}$, then

$$
\begin{aligned}
\max w_{i}-\min w_{i} & =w_{i_{0}}-w_{j_{0}} \\
& =\left|w_{i_{0}}-w_{j_{0}}\right| \\
& \leq \max _{i \in\{1, \cdots, n-1\}, j \in\left\{i_{0}+1, \cdots, n\right\}}\left|w_{i}-w_{j}\right|
\end{aligned}
$$

If $i_{0}>j_{0}$, then

$$
\begin{aligned}
\max w_{i}-\min w_{i} & =w_{i_{0}}-w_{j_{0}} \\
& =\left|w_{j_{0}}-w_{i_{0}}\right| \\
& \leq \max _{i \in\{1, \cdots, n-1\}, j \in\left\{j_{0}+1, \cdots, n\right\}}\left|w_{i}-w_{j}\right| .
\end{aligned}
$$

and hence the equality holds. 
Then the corresponding extended minimax disparity model for RIM quantifier problem with given orness level can be proposed as follows:

$$
\begin{array}{ll}
\text { Minimize } & \operatorname{ess} \sup f-\operatorname{ess} \inf f \\
\text { subject to } & \int_{0}^{1} r f(r) d r=1-\alpha, 0<\alpha<1, \\
& \int_{0}^{1} f(r) d r=1, f(r) \geq 0 .
\end{array}
$$

\section{Relation of Solutions between OWA Operator Model and RIM Quantifier Model}

The following result is the solution of the extended minimax OWA operator problem given by Hong [26].

Theorem 1 ( $n=2 k$ :even). An optimal weight for the constrained optimization problem (2) for a given level of $\alpha=\operatorname{orness}(W)$ should satisfy the following equation:

$$
\begin{gathered}
H(\alpha)=\text { Minimize }\left\{\max _{i \in\{1, \cdots, n-1\}, j \in\{i+1, \cdots, n\}}\left|w_{i}-w_{j}\right|\right\}=\left|\frac{(1-2 \alpha)(n-1)}{(n-m) m}\right| \\
w_{1}^{*}=w_{2}^{*}=\cdots=w_{m}^{*}, w_{k+1}^{*}=w_{k+2}^{*}=\cdots=w_{n^{\prime}}^{*}
\end{gathered}
$$

where

$$
w_{1}^{*}=\frac{m-(1-2 \alpha)(n-1)}{n m}
$$

and

$$
w_{m+1}^{*}=\frac{n-m-(2 \alpha-1)(n-1)}{n(n-m)} .
$$

Here $m$ satisfies the following:

$$
m= \begin{cases}\lceil(1-2 \alpha)(n-1)\rceil, & \text { if } 0 \leq \alpha \leq \frac{n-2}{4(n-1)}, \\ k, & \text { if } \frac{n-2}{4(n-1)} \leq \alpha \leq \frac{3 n-2}{4(n-1)}, \\ n-\lceil(2 \alpha-1)(n-1)\rceil, & \text { if } \frac{3 n-2}{4(n-1)} \leq \alpha \leq 1 .\end{cases}
$$

where $\lceil x\rceil=m+1 \Longleftrightarrow m<x \leq m+1$ for any integer $m$.

Can we get a hint about the solution of the extended minimax Rim quantifier problem? Here, we suggest an idea.

For a given associated weighting vector $W_{n}=\left(w_{1}, \cdots, w_{n}\right)$ of having the property $w_{1}+\cdots+w_{n}=1,0 \leq w_{i} \leq 1, i=1, \cdots, n$, we define a generating function $f(t)$

$$
f_{W_{n}}(x)=n w_{i}, \quad x \in\left[\frac{i}{n}, \frac{i+1}{n}\right), i=0,1, \cdots, n-1,
$$

having the property $\int_{0}^{1} f_{W}^{n}(x) d x=1$ and let

$$
f^{*}(x)=\lim _{n \rightarrow \infty}=f_{W_{n}}(x) .
$$

Can this function $f^{*}(x)$ be a solution of the corresponding extended minimax Rim quantifier problem? Maybe, yes! Let's try to follow this idea. 
For given $W_{n}^{*}=\left(w_{1}^{*}, \cdots, w_{n}^{*}\right)$ from above Theorem 1 , we have for $0<\alpha \leq \frac{1}{4}$,

$$
f_{\mathrm{W}_{n}^{*}}(x)=\left\{\begin{array}{lll}
\frac{\lceil(1-2 \alpha)(n-1)\rceil-(1-2 \alpha)(n-1)}{\lceil(1-2 \alpha)(n-1)\rceil}, & \text { if } & x \in\left[0, \frac{\lceil(1-2 \alpha)(n-1)\rceil}{n}\right) \\
\frac{n-\lceil(1-2 \alpha)(n-1)\rceil-(2 \alpha-1)(n-1)}{n-\lceil(1-2 \alpha)(n-1)\rceil}, & \text { if } & x \in\left[\frac{\lceil(1-2 \alpha)(n-1)\rceil}{n}, 1\right] .
\end{array}\right.
$$

for $\frac{1}{4} \leq \alpha \leq \frac{3}{4}$

$$
f_{W_{n}^{*}}(x)=\left\{\begin{array}{lll}
\frac{n / 2-(1-2 \alpha)(n-1)}{n / 2}, & \text { if } & x \in\left[0, \frac{1}{2}\right) \\
\frac{n / 2-(2 \alpha-1)(n-1)}{(n / 2)}, & \text { if } & x \in\left[\frac{1}{2}, 1\right] .
\end{array}\right.
$$

for $3 / 4 \leq \alpha \leq 1$,

$$
f_{W_{n}^{*}}(x)=\left\{\begin{array}{lll}
\frac{n-\lceil(2 \alpha-1)(n-1)\rceil-(1-2 \alpha)(n-1)}{n-\lceil(2 \alpha-1)(n-1)\rceil}, & \text { if } & x \in\left[0,1-\frac{\lceil(1-2 \alpha)(n-1)\rceil}{n}\right) \\
\frac{\lceil(2 \alpha-1)(n-1)\rceil-(2 \alpha-1)(n-1)}{\lceil(2 \alpha-1)(n-1)\rceil} & \text { if } & x \in\left[1-\frac{\lceil(1-2 \alpha)(n-1)\rceil}{n}, 1\right] .
\end{array}\right.
$$

Let $\lim _{n \rightarrow \infty} f_{W_{n}^{*}}(x)=f^{*}(x)$, then

1. for $0<\alpha \leq \frac{1}{4}$,

$$
f^{*}(r)=\left\{\begin{array}{lll}
0, & \text { if } & r \in[0,1-2 \alpha) \\
\frac{1}{2 \alpha}, & \text { if } & r \in[1-2 \alpha, 1] .
\end{array}\right.
$$

2. for $\frac{1}{4} \leq \alpha \leq \frac{3}{4}$,

$$
f^{*}(r)=\left\{\begin{array}{lll}
4 \alpha-1, & \text { if } & r \in\left[0, \frac{1}{2}\right) \\
3-4 \alpha, & \text { if } & r \in\left[\frac{1}{2}, 1\right]
\end{array}\right.
$$

3. for $\frac{3}{4}<\alpha \leq 1$,

$$
f^{*}(r)= \begin{cases}\frac{1}{2(1-\alpha)}, & \text { if } \quad r \in[0,2 \alpha] \\ 0, & \text { elsewhere }\end{cases}
$$

In the following section, we will show that $f^{*}$ can be the solution of the extended minimax RIM quantifier problem.

\section{Proof of the Extended Minimax RIM Quantifier Problem}

In this section, we prove the following main result.

Theorem 2. The optimal solution for problem (2) for given orness level $\alpha$ is the weighting function $f^{*}$ such that

1. for $0<\alpha \leq \frac{1}{4}$,

$$
f^{*}(r)=\left\{\begin{array}{lll}
0 \text { a.e., } & \text { if } & r \in[0,1-2 \alpha) \\
\frac{1}{2 \alpha} \text { a.e., } & \text { if } & r \in[1-2 \alpha, 1] .
\end{array}\right.
$$

2. for $\frac{1}{4} \leq \alpha \leq \frac{3}{4}$,

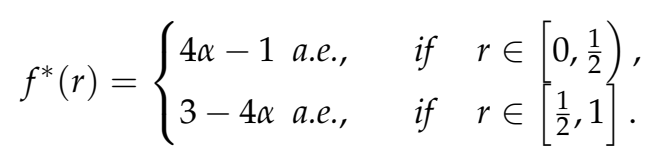


3. for $\frac{3}{4}<\alpha \leq 1$,

$$
f^{*}(r)= \begin{cases}\frac{1}{2(1-\alpha)} \text { a.e., } & \text { if } r \in[0,2 \alpha] \\ 0 \text { a.e., } & \text { elsewhere. }\end{cases}
$$

and

$$
H(\alpha)=\text { Minimize } \mid \text { ess supf }-\operatorname{ess} \inf f \mid= \begin{cases}\frac{1}{2 \alpha} & \text { if } 0<\alpha \leq \frac{1}{4} \\ 4|(1-2 \alpha)| & \text { if } \frac{1}{4} \leq \alpha \leq \frac{3}{4} \\ \frac{1}{2 \alpha} & \text { if } \frac{3}{4}<\alpha \leq 1\end{cases}
$$

We need the following two lemma's to prove the main result. We denote $D_{f}(x)=\int_{0}^{x} f(t) d t$, $0 \leq x \leq 1$ and $E(f)=\int_{0}^{1} r f(r) d r$.

The following result is known.

Lemma 1. $E(f)=\int_{0}^{1}\left(1-D_{f}(t)\right) d t$.

Lemma 2. Let ess inf $f=\beta_{0} \geq 0$ and ess sup $f=\beta_{1}>0$ such that $\int_{0}^{1} f(r) d r=1$ and define a function $f_{0}$ as

$$
f_{0}(r)= \begin{cases}\beta_{0} \text { a.e., } & \text { if } \quad r \in\left[0, c_{0}\right), \\ \beta_{1} \text { a.e., } & \text { if } \quad r \in\left[c_{0}, 1\right] .\end{cases}
$$

for some $c_{0} \in(0,1)$ such that $\int_{0}^{1} f_{0}(r) d r=1$. Then we have $E(f) \leq E\left(f_{0}\right)$ and the equality holds iff $f=f_{0}$ a.e.

Proof. The result follows immediately from Lemma 1 if we show that $D_{f_{0}}(x) \leq D_{f}(x), x \in[0,1]$. It is clear that $D_{f_{0}}(x) \leq D_{f}(x), x \in\left[0, c_{0}\right]$. Suppose that there exists a point $t_{0} \in\left(c_{0}, 1\right)$ such that $D_{f_{0}}\left(t_{0}\right)>D_{f}\left(t_{0}\right)$. Then

$$
\int_{t_{0}}^{1} \beta_{1} d r=\int_{t_{0}}^{1} f_{0}(r) d r=1-D_{f_{0}}\left(t_{0}\right)<1-D_{f}\left(t_{0}\right)=\int_{t_{0}}^{1} f(r) d r
$$

which implies $\operatorname{ess} \sup _{\left(t_{0}, 1\right)} f>\beta_{1}$. It is a contradiction.

Proof of Theorem 2. If $\alpha=\frac{1}{2}$, we clearly have the optimal solution is $f^{*}(r)=1$ a.e. for $r \in[0,1]$. Note that ess inf $f^{*}<1<\operatorname{ess} \sup f^{*}$ for $\alpha \in\left(0, \frac{1}{2}\right)$. Without loss of generality, we can assume that $\alpha \in\left(0, \frac{1}{2}\right)$, since if a weighting function $f^{*}(r)$ is optimal to problem (2) for some given level of preference $\alpha \in\left(0, \frac{1}{2}\right]$, then $f^{*}(1-r)$ is optimal to the problem (2) for a given level of preference $1-\alpha$. Indeed, since $D_{f}=D_{f^{R}}, \int_{0}^{1} f(r) d r=\int_{0}^{1} f^{R}(r) d r$ and $E\left(f^{R}\right)=1-E(f)$, where $f^{R}(r)=f(1-r)$ hence for $\alpha>\frac{1}{2}$, we can consider problem (2) for the level of preference with index $1-\alpha$, and then take the reverse of that optimal solution. We can easily check that the weighting functions, $f^{*}$, given above are feasible for problem (2). We show that $f^{*}$ is the unique optimal solution for a given $\alpha$. Let nonnegative function $f$ satisfy $1=\int_{0}^{1} f(r) d r$ and $E(f)=\int_{0}^{1} r f(r) d r=1-\alpha$. Let ess inf $f=\beta_{0}$ and ess sup $f=\beta_{1}$.

Case (A): $\alpha \in\left(0, \frac{1}{4}\right]$. 
We note that $\operatorname{ess} \inf f^{*}-$ ess $\inf f^{*}=\frac{1}{2 \alpha}$. We will show that $\beta_{1}-\beta_{0} \geq \frac{1}{2 \alpha}$. To show this, we define a function $f_{0}$ as

$$
f_{0}(r)=\left\{\begin{array}{lll}
\beta_{0} & \text { if } & r \in\left[0, x_{0}\right) \\
\beta_{1} & \text { if } & r \in\left[x_{0}, 1\right]
\end{array}\right.
$$

for some $x_{0} \in(0,1)$ such that $\int_{0}^{1} f_{0}(r) d r=1$. Then by Lemma 2, $E(f) \leq E\left(f_{0}\right)$. Suppose that $\beta_{1}-\beta_{0}<\frac{1}{2 \alpha}$ and define another function $f_{0}^{*}$ as

$$
f_{0}^{*}(r)=\left\{\begin{array}{lll}
\beta_{0} & \text { if } & r \in\left[0, x_{0}^{*}\right) \\
\beta_{0}+\frac{1}{2 \alpha} & \text { if } & r \in\left[x_{0}^{*}, 1\right]
\end{array}\right.
$$

for some $x_{0}^{*} \in(0,1)$ such that $\int_{0}^{1} f_{0}^{*}(r) d r=1$. Then $E\left(f_{0}\right)<E\left(f_{0}^{*}\right)$. We note that $1=\beta_{0} x_{0}^{*}+(1-$ $\left.x_{0}^{*}\right)\left(\beta_{0}+\frac{1}{2 \alpha}\right)$. Then

$$
x_{0}^{*}=2 \alpha \beta_{0}+1-2 \alpha .
$$

We know that

$$
\begin{aligned}
E\left(f_{0}^{*}\right) & =\beta_{0} \int_{0}^{x_{0}^{*}} x d x+\left(\beta_{0}+\frac{1}{2 \alpha}\right) \int_{x_{0}^{*}}^{1} x d x \\
& =\frac{\beta_{0}}{2}+\frac{1}{4 \alpha}-\frac{x_{0}^{* 2}}{4 \alpha}
\end{aligned}
$$

and

$$
E\left(f^{*}\right)=\frac{1}{2 \alpha} \int_{1-2 \alpha}^{1} x d x=1-\alpha
$$

And we have

$$
\begin{aligned}
E\left(f^{*}\right)-E\left(f_{0}^{*}\right) & =\frac{1}{2} \frac{x_{0}^{* 2}}{2 \alpha}-\frac{1}{2} \frac{(1-2 \alpha)^{2}}{2 \alpha}-\frac{\beta_{0}}{2} \\
& =\frac{1}{2}\left[\frac{1}{2 \alpha} x_{0}^{* 2}-\frac{(1-2 \alpha)^{2}}{2 \alpha}-\beta_{0}\right] \\
& =\frac{1}{2}\left[\frac{1}{2 \alpha}\left(2 \alpha \beta_{0}+1-2 \alpha\right)^{2}-\frac{(1-2 \alpha)^{2}}{2 \alpha}-\beta_{0}\right] \\
& =\frac{\beta_{0}}{2}\left[2 \alpha \beta_{0}+2(1-2 \alpha)-1\right] \\
& \geq 0
\end{aligned}
$$

where the third equality comes from (3) and the last inequality comes from the facts that $1-2 \alpha \geq \frac{1}{2}$, $\beta_{0} \geq 0$ and $\alpha>0$. This proves $E(f)<E\left(f_{0}^{*}\right) \leq E\left(f^{*}\right)=1-\alpha$, which is a contradiction. Hence $f^{*}$ is an optimal solution for the case of $\alpha \in\left(0, \frac{1}{4}\right]$.

Case (B): $\alpha \in\left(\frac{1}{4}, \frac{1}{2}\right)$.

We note that ess inf $f^{*}-$ ess inf $f^{*}=4(1-2 \alpha)$. We will show that $\beta_{1}-\beta_{0} \geq 4(1-2 \alpha)$. As in the Case (A), we define a function $f_{0}$ as

$$
f_{0}(r)=\left\{\begin{array}{lll}
\beta_{0} & \text { if } & r \in\left[0, x_{0}\right), \\
\beta_{1} & \text { if } & r \in\left[x_{0}, 1\right],
\end{array}\right.
$$


for some $x_{0} \in(0,1)$ such that $\int_{0}^{1} f_{0}(r) d r=1$. Then by lemma $2, E(f) \leq E\left(f_{0}\right)$. Suppose that $\beta_{1}-\beta_{0}<\frac{1}{2 \alpha}$ and define another function $f_{1}^{*}$ as

$$
f_{1}^{*}(r)=\left\{\begin{array}{lll}
\beta_{0} & \text { if } & r \in\left[0, x_{1}^{*}\right), \\
\beta_{0}+4(1-2 \alpha) & \text { if } & r \in\left[x_{1}^{*}, 1\right],
\end{array}\right.
$$

for some $x_{1}^{*} \in(0,1)$ such that $\int_{0}^{1} f_{1}^{*}(r) d r=1$. Then, since $x_{0}<x_{1}^{*}$, by lemma $2 E\left(f_{0}\right)<E\left(f_{1}^{*}\right)$. We note that $1=\beta_{0} x_{1}^{*}+\left(1-x_{1}^{*}\right)\left(\beta_{0}+4(1-2 \alpha)\right)$. Then

$$
x_{1}^{*}=1+\frac{\beta_{0}-1}{4(1-2 \alpha)}
$$

and

$$
x_{1}^{* 2}=1+\frac{\beta_{0}-1}{2(1-2 \alpha)}+\frac{\left(\beta_{0}-1\right)^{2}}{16(1-2 \alpha)^{2}}
$$

We know that

$$
\begin{aligned}
E\left(f_{0}^{*}\right) & =\beta_{0} \int_{0}^{x_{1}^{*}} x d x+\left(\beta_{0}+4(1-2 \alpha)\right) \int_{x_{1}^{*}}^{1} x d x \\
& =\frac{1}{2}\left[\beta_{0}+4(1-2 \alpha)\right]-2(1-2 \alpha) x_{1}^{* 2}
\end{aligned}
$$

and

$$
\begin{aligned}
E\left(f^{*}\right) & =(4 \alpha-1) \int_{0}^{\frac{1}{2}} x d x+(3-4 \alpha) \int_{\frac{1}{2}}^{1} x d x \\
& =1-\alpha .
\end{aligned}
$$

Then we have that

$$
\begin{aligned}
E\left(f^{*}\right)-E\left(f_{1}^{*}\right) & =3 \alpha-1-\frac{\beta_{0}}{2}+2(1-2 \alpha) x_{1}^{* 2} \\
& =\frac{\left(\beta_{0}-1\right)^{2}}{8(1-2 \alpha)}+\frac{\beta_{0}}{2}-\alpha \\
& =\frac{\left[\beta_{0}-(4 \alpha-1)\right]^{2}}{8(1-2 \alpha)} \\
& \geq 0
\end{aligned}
$$

where the second equality comes from (4) and hence $E(f)<E\left(f_{1}^{*}\right) \leq E\left(f^{*}\right)=1-\alpha$, which is a contradiction. This completes the proof.

\section{Conclusions}

Previous studies have suggested a number of methods for obtaining optimal solution of the RIM quantifier problem. This paper proposes the extended minimax disparity RIM quantifier problem under a given orness level. We completely prove it analytically.

Funding: This research was supported by Basic Science Research Program through the National 247 Research Foundation of Korea (NRF) funded by the Ministry of Education (2017R1D1A1B03027869).

Conflicts of Interest: The authors declare no conflict of interest. 


\section{References}

1. Amin, G.R.; Emrouznejad, A. An extended minimax disparity to determine the OWA operator weights. Comput. Ind. Eng. 2006, 50, 312-316. [CrossRef]

2. Amin, G.R. Notes on priperties of the OWA weights determination model. Comput. Ind. Eng. 2007, 52, 533-538. [CrossRef]

3. Emrouznejad, A.; Amin, G.R. Improving minimax disparity model to determine the OWA operator weights. Inf. Sci. 2010, 180, 1477-1485. [CrossRef]

4. Emrouznejad, A. MP-OWA: The most preferred OWA operator. Knowl. Based Syst. 2008, 21, 847-851. [CrossRef]

5. Emrouznejad, A.; Marra, M. Ordered Weighted Averaging Operators 1988-2014: A citation-based literature survey. Int. J. Intell. Syst. 2014, 29, 994-1014. [CrossRef]

6. Filev, D.; Yager, R.R. On the issue of obtaining OWA operator weights. Fuzzy Sets Syst. 1988, 94, 157-169. [CrossRef]

7. Fullér, R.; Majlender, P. An analytic approach for obtaining maximal entropy OWA operators weights. Fuzzy Sets Syst. 2001, 124, 53-57. [CrossRef]

8. Fullér, R.; Majlender, P. On obtaining minimal variability OWA operator weights. Fuzzy Sets Syst. 2003, 136, 203-215. [CrossRef]

9. O'Hagan, M. Aggregating template or rule antecedents in real-time expert systems with fuzzy set logic. In Proceedings of the 22nd annual IEEE Asilomar Conf. on Signals, Systems, Computers, Pacific Grove, CA, USA, 31 October-2 November 1988; pp. 681-689.

10. Hong, D.H. A note on the minimal variability OWA operator weights. Int. J. Uncertainty, Fuzziness Knowl. Based Syst. 2006, 14, 747-752. [CrossRef]

11. Hong, D.H. A note on solution equivalence to general models for RIM quantifier problems. Fuzzy Sets Syst. 2018, 332, 25-28. [CrossRef]

12. Wheeden, R.L.; Zygmund, A. Measure and Integral: An Introduction to Real Analysis; Marcel Dekker, Inc.: New York, NY, USA, 1977.

13. Wang, Y.M.; Parkan, C. A minimax disparity approach obtaining OWA operator weights. Inf. Sci. 2005, 175, 20-29. [CrossRef]

14. Wang, Y.M.; Luo, Y.; Liu, X. Two new models for determining OWA operater weights. Comput. Ind. Eng. 2007, 52, 203-209. [CrossRef]

15. Yager, R.R. Ordered weighted averaging aggregation operators in multi-criteria decision making. IEEE Trans. Syst. Man Cybern. 1988, 18, 183-190. [CrossRef]

16. Yager, R.R. OWA aggregation over a continuous interval argument with application to decision making. IEEE Trans. Syst. Man Cybern. Part B 2004, 34, 1952-1963. [CrossRef]

17. Yager, R.R. Families of OWA operators. Fuzzy Sets Syst. 1993, 59, 125-148. [CrossRef]

18. Yager, R.R.; Filev, D. Induced ordered weighted averaging operators. IEEE Trans. Syst. Man Cybern. Part B Cybern. 1999, 29, 141-150. [CrossRef]

19. Liu, X. On the maximum entropy parameterized interval approximation of fuzzy numbers. Fuzzy Sets Syst. 2006, 157, 869-878. [CrossRef]

20. Liu, X.; Da, Q. On the properties of regular increasing monotone (RIM) quantifiers with maximum entropy. Int. J. Gen. Syst. 2008, 37, 167-179. [CrossRef]

21. Liu, X.; Lou, H. On the equivalence of some approaches to the OWA operator and RIM quantifier determination. Fuzzy Sets Syst. 2007, 159, 1673-1688. [CrossRef]

22. Hong, D.H. The relationship between the minimum variance and minimax disparity RIM quantifier problems. Fuzzy Sets Syst. 2011, 181, 50-57. [CrossRef]

23. Hong, D.H. The relationship between the maximum entropy and minimax ratio RIM quantifier problems. Fuzzy Sets Syst. 2012, 202, 110-117. [CrossRef]

24. Liu, X. A general model of parameterized OWA aggregation with given orness level. Int. J. Approx. Reason. 2008, 48, 598-627. [CrossRef] 
25. Hong, D.H. The general model for least square disparity RIM quantifier problems. Fuzzy Optim. Decis. Mak. 2019, submitted.

26. Hong, D.H. On proving the extended minimax disparity OWA problem. Fuzzy Sets Syst. 2011, 168, 35-46. [CrossRef]

(c)

(C) 2019 by the authors. Licensee MDPI, Basel, Switzerland. This article is an open access article distributed under the terms and conditions of the Creative Commons Attribution (CC BY) license (http:/ / creativecommons.org/licenses/by/4.0/). 